Article

\title{
An Extremely Efficient Boundary Element Method for Wave Interaction with Long Cylindrical Structures Based on Free-Surface Green's Function
}

\author{
Yingyi Liu ${ }^{1, *}$, Ying Gou ${ }^{2}$, Bin Teng ${ }^{2}$ and Shigeo Yoshida ${ }^{1}$ \\ 1 Renewable Energy Center, Research Institute for Applied Mechanics, Kyushu University, \\ Kasuga 8168580, Japan; yoshidas@riam.kyushu-u.ac.jp \\ 2 State Key Laboratory of Coastal and Offshore Engineering, Dalian University of Technology, \\ Dalian 116024, China; gouying@dlut.edu.cn (Y.G.); bteng@dlut.edu.cn (B.T.) \\ * Correspondence: liuyingyi@riam.kyushu-u.ac.jp; Tel.: +81-80-8565-7934 \\ Academic Editor: Qinjun Kang \\ Received: 8 August 2016; Accepted: 12 September 2016; Published: 16 September 2016
}

\begin{abstract}
The present study aims to develop an efficient numerical method for computing the diffraction and radiation of water waves with horizontal long cylindrical structures, such as floating breakwaters in the coastal region, etc. A higher-order scheme is used to discretize geometry of the structure as well as the physical wave potentials. As the kernel of this method, Wehausen's free-surface Green function is calculated by a newly-developed Gauss-Kronrod adaptive quadrature algorithm after elimination of its Cauchy-type singularities. To improve its computation efficiency, an analytical solution is derived for a fast evaluation of the Green function that needs to be implemented thousands of times. In addition, the OpenMP parallelization technique is applied to the formation of the influence coefficient matrix, significantly reducing the running CPU time. Computations are performed on wave-exciting forces and hydrodynamic coefficients for the long cylindrical structures, either floating or submerged. Comparison with other numerical and analytical methods demonstrates a good performance of the present method.
\end{abstract}

Keywords: long cylindrical structure; free-surface Green function; higher-order boundary element method; multipole expansion; singularity elimination; Gauss-Kronrod; numerical quadrature; OpenMP parallelization

\section{Introduction}

Cylindrical structures have been widely used in the rapidly-developing coastal and offshore engineering industries in recent decades, in the form of such as floating breakwaters, oscillating water columns (OWC) for power generation, etc. These devices are used to either passively avoid the large wave kinematic energy from attacking harbors or actively convert the wave energy into other kinds of energies. Cylindrical structures are important in the industries probably due to their simplicity in geometry and the relatively lower fluid forces they may experience. Extensive efforts have been made on investigation of such kind of important structures, theoretically [1-3], numerically [4-7], and experimentally $[8,9]$. In the numerical approaches, boundary element method should be one of the most popular tools for analysis [5-7]. However, the present work is very different from the classical research in the following aspects: (1) since the governing equation used herein is the Laplace equation instead of the Helmholtz equation, Wehausen's free-surface Green function [10], constituted by several simple arithmetic functions, can be employed instead of Haskind's Green function $[5,11]$ which may require many evaluations of the modified Bessel functions; (2) since the free-surface Green function is used as the kernel instead of the Rankine Green function, meshing of the geometry could be restricted to only the body surface, such that there is no need to deal with the open boundaries, as has been 
accomplished by Zheng et al. [7]; (3) application of a three-node higher-order element rather than a traditional constant or linear element guarantees the accuracy of geometrical/physical discretization; and (4) thanks to the exponential integral functions, the free-surface Green function could be written in a simpler form and then evaluated in a faster speed with a precise result. All of the above advantages facilitate numerical investigations for hydrodynamic performances of such cylindrical structures.

These horizontal cylindrical structures are so long in its axis direction that the problem to be solved could be considered in two-dimensional. In comparison to the three-dimensional wave-structure interaction model within the framework of linear potential flow theory, the present simplified two-dimensional model has many fewer unknowns on the body surface, since the surface integrations have been substituted by line integrations. In our HOBEM (higher-order boundary element method) model, for a typical frequency domain problem, about 10 50 elements (or, in other words, less than 100 nodes) are sufficient to represent an arbitrary cross-sectional shape. Therefore, generally about $10^{2} \sim 10^{4}$ evaluations of the Green function are needed for each incident wave period, compared to those $\mathcal{O}\left(10^{6}\right)$ evaluations in the three-dimensional cases (see [12]). Furthermore, by taking advantage of the contemporary computational technologies, some special technique may be applied to parallelize the algorithm on multi-processor machines.

Apart from the HOBEM discretization, efficient evaluation of the free-surface Green function is another important issue in this work. Numerous studies have been performed in the field since 1980s. Noblesse and Newman have made the most important contributions for this issue [12-17]. They developed several popular methods, e.g., separating the local component from the far-field one and then calculate them by tabulation algorithm, or making the singular functions slow-varying by subtracting some component and then approximate the resulting functions by Chebyshev approximation. These methods have been simplified in the present model since the problem to be considered is two-dimensional and in infinite water depth, in which an extremely convenient analytical solution can be found for the Green's kernel in the boundary integral equation. The numerical results in Section 3 show the validity and efficiency of the present method.

\section{Mathematical Theory and Algorithms}

\subsection{Governing Equation and Boundary Conditions}

The problem is to consider interactions between linear water waves and a long prismatic rigid structure in arbitrary cross-sectional shape, either floating or submerged in water of infinite depth, as shown in Figure 1. The right-handed Cartesian coordinate system $(x, z)$ is defined, with its origin located at the undisturbed free surface level and the $z$-axis taken vertically upward. The fluid domain is denoted by $\Omega$, whose boundaries $S$ consists of a free surface boundary $S_{\mathrm{F}}$, an up-side open boundary $S_{\mathrm{U}}$, a lee-side open boundary $S_{\mathrm{L}}$, and a wetted surface boundary $S_{\mathrm{B}}$ on the structure.

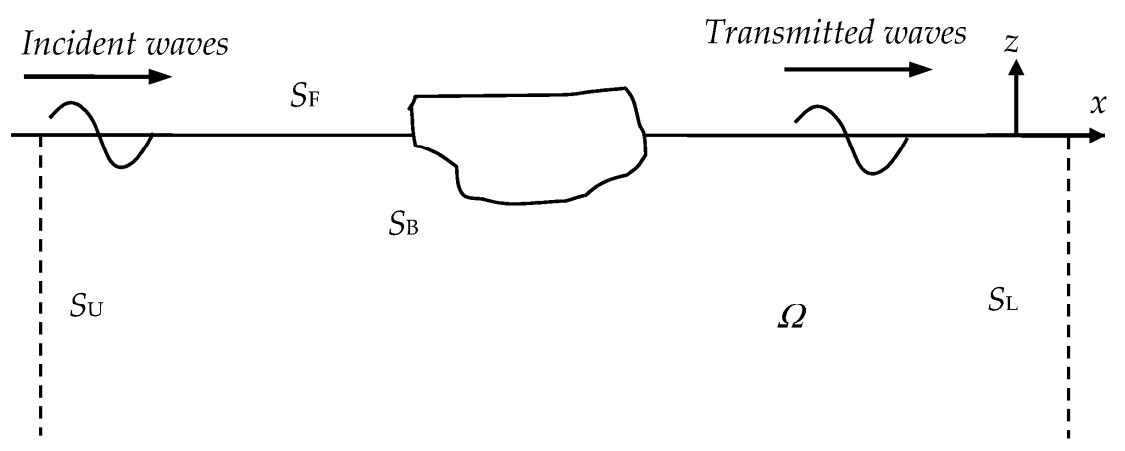

Figure 1. Computation domain of the problem. 
The fluid is assumed to be inviscid and incompressible, and the motion is assumed irrotational. For the linear small amplitude wave harmonic in time with an angular frequency $\omega$, the velocity potential can be expressed by:

$$
\Phi(x, z, t)=\operatorname{Re}\left[\phi(x, z, t) e^{-\mathrm{i} \omega t}\right]
$$

where $\phi$ is a time-independent complex velocity potential, which can be further decomposed into:

$$
\phi=\phi_{0}+\phi_{4}-\mathrm{i} \omega \sum_{j=1}^{3} \chi_{j} \phi_{j}
$$

where the three components denote incident potential, diffraction potential, and radiation potential, respectively; $\chi_{j}$ represents displacement of the body motion in each mode (sway, heave, or roll), and $\phi_{j}$ stands for the corresponding radiation potential to each motion mode.

The incoming wave of amplitude $A$ and frequency $\omega$, propagating in the positive $x$ direction in the water of infinite water depth, can be described by the following incident velocity potential:

$$
\phi_{0}=-\frac{\mathrm{i} g A}{\omega} e^{K z+\mathrm{i} K x}
$$

where $K$ is the infinite depth wave number defined by $K=\omega^{2} / g$. The four induced wave potentials $\phi_{j}(j=1 \sim 4)$ must satisfy the Laplace equation:

$$
\nabla^{2} \phi_{j}=0
$$

and be subjected to various boundary conditions in the fluid domain, including the free surface condition on $S_{\mathrm{F}}$ :

$$
\frac{\partial \phi_{j}}{\partial z}-K \phi_{j}=0
$$

the bottom boundary condition as $z \rightarrow \infty$ :

$$
\nabla \phi_{j} \rightarrow 0
$$

the boundary condition on the surface $S_{\mathrm{B}}$ of the structure:

$$
\frac{\partial \phi_{j}}{\partial \mathrm{n}}=\left\{\begin{array}{lr}
-\frac{\partial \phi_{0}}{\partial \mathrm{n}}, & (j=4) \\
n_{j}, & (j=1,2,3)
\end{array},\right.
$$

and the radiation condition in the far field boundaries $S_{U}$ and $S_{\mathrm{L}}$ :

$$
\lim _{x \rightarrow \pm \infty}\left(\frac{\partial \phi_{j}}{\partial x} \pm \mathrm{i} K \phi_{j}\right)=0,
$$

where $n$ is the normal direction of the body geometry, with its three components $n_{1}=n_{\mathrm{x}}, n_{2}=n_{\mathrm{z}}$, $n_{3}=\left(z-z_{\mathrm{c}}\right) n_{\mathrm{x}}-\left(x-x_{\mathrm{c}}\right) n_{\mathrm{z}}$, where $n_{\mathrm{x}}$ and $n_{\mathrm{z}}$ are the $x$ and $z$ components of the unit inward normal, respectively, and $\left(x_{\mathrm{c}}, z_{\mathrm{c}}\right)$ is the rotation center. The subscripts $j=1,2,3$ denote the direction of sway, heave, and roll for radiation, respectively, and $j=4$ stands for the diffraction. 


\subsection{Numerical Techniques}

According to Green's second theorem, by employing Wehausen's free-surface Green function as the kernel, a boundary integral equation can be obtained as:

$$
\alpha \phi_{j}\left(\mathbf{x}_{0}\right)=\int_{S_{B}}\left[\frac{\partial G\left(\mathbf{x} ; \mathbf{x}_{0}\right)}{\partial \mathrm{n}} \phi_{j}(\mathbf{x})-G\left(\mathbf{x} ; \mathbf{x}_{0}\right) \frac{\partial \phi_{j}(\mathbf{x})}{\partial \mathrm{n}}\right] d S,
$$

where $\alpha$ is the solid angle. The free-surface Green function is defined to be:

$$
G\left(\mathbf{x} ; \mathbf{x}_{0}\right)=\ln \frac{r}{r_{1}}-2 \oint_{0}^{\infty} \frac{e^{\mu(z+\zeta)}}{\mu-K} \cos \mu(x-\xi) d \mu,
$$

where the path of the contour integral passes below the poles at $\mu=K$; coordinate of the source point is $\mathbf{x}_{0}=(\xi, \zeta) ; r$ is the distance between field point and source point, and $r_{1}$ is the distance between field point and the image of source point with respect to the free surface.

On the other aspect, if Rankine Green function were employed as the kernel, the boundary integral equation would be:

$$
\alpha \phi_{j}\left(\mathbf{x}_{0}\right)=\int_{S_{F}+S_{B}+S_{U}+S_{L}}\left[\frac{\partial G\left(\mathbf{x} ; \mathbf{x}_{0}\right)}{\partial \mathrm{n}} \phi_{j}(\mathbf{x})-G\left(\mathbf{x} ; \mathbf{x}_{0}\right) \frac{\partial \phi_{j}(\mathbf{x})}{\partial \mathrm{n}}\right] d S,
$$

where the kernel would be simply expressed by:

$$
G\left(\mathbf{x} ; \mathbf{x}_{0}\right)=\ln r .
$$

In this paper, we denote the method based on Equations (9) and (10) as FSG_BEM, and the method based on Equations (11) and (12) as RKG_BEM. The former is applied as the present numerical method, while the latter is used as a comparison for computational efficiency.

The three-node isoparametric element is selected to discretize both the geometry of body surface and the physical variables, the shape functions of which being expressed by:

$$
\begin{gathered}
h_{1}(\eta)=\frac{1}{2} \eta(\eta-1), \\
h_{2}(\eta)=1-\eta^{2}, \\
h_{3}(\eta)=\frac{1}{2} \eta(\eta+1),
\end{gathered}
$$

where $\eta$ is the local coordinate $(-1 \leq \eta \leq 1)$. Therefore, the velocity potential and its normal derivative on the boundary surface can be expressed straightforwardly as:

$$
\begin{gathered}
{[x, z]=\sum_{k=1}^{3} h_{k}(\eta)\left[x^{k}, z^{k}\right],} \\
{\left[\phi, \frac{\partial \phi}{\partial \mathrm{n}}\right]=\sum_{k=1}^{3} h_{k}(\eta)\left[\phi^{k},\left(\frac{\partial \phi}{\partial \mathrm{n}}\right)^{k}\right] .}
\end{gathered}
$$


Applying the above discretization and the body surface condition Equations (5)-(8) leads to the following discrete form of the boundary integral equation of Equation (9):

$$
\begin{aligned}
& \alpha \phi_{j}\left(\mathbf{x}_{0}\right)-\sum_{i=1}^{N_{B}} \int_{-1}^{1} \frac{\partial G\left(\mathbf{x} ; \mathbf{x}_{0}\right)}{\partial \mathrm{n}} \sum_{k=1}^{3} h_{k}(\eta)\left(\phi_{j}\right)^{k}|J(\eta)| d \eta \\
& =\left\{\begin{array}{l}
\sum_{i=1}^{N_{B}} \int_{-1}^{1} G\left(\mathbf{x} ; \mathbf{x}_{0}\right) \frac{\partial \phi_{0}(\mathbf{x})}{\partial \mathrm{n}}|J(\eta)| d \eta, \quad(j=4), \\
-\sum_{i=1}^{N_{B}} \int_{-1}^{1} G\left(\mathbf{x} ; \mathbf{x}_{0}\right) n_{j}|J(\eta)| d \eta, \quad(j=1,2,3)
\end{array}\right.
\end{aligned}
$$

where $N_{B}$ represents the number of total elements along the body surface, and $J(\eta)$ the Jacobi matrix for local-global coordinate transformation, the determinant value of which is calculated by:

$$
|J(\eta)|=\sqrt{\left(\frac{d x}{d \eta}\right)^{2}+\left(\frac{d z}{d \eta}\right)^{2}} .
$$

By employing a collocation process for Equation (18) that the source point is arranged to be put on each grid node on the immersed body surface mesh, a linear algebraic system could be obtained in closed form:

$$
[A]_{N \times N}\left[\phi_{j}\right]_{N \times N}=[B]_{N \times 4}
$$

where $N$ is the total number of nodes. Solution of the above linear system is sensitive to the diagonal terms of the left-hand side influence matrix $[A]$ which, therefore, needs to be evaluated precisely with caution. However, direct calculation of these diagonal terms is usually inaccurate and troublesome, due to the high singularity of the Green function in the case when the field point and the source point coincides with each other. Fortunately, this weakness can be avoided by considering a constant flux across the fluid $(\phi=1)$; thereafter we obtain:

$$
A_{i i}=-\sum_{j=1, j \neq i}^{N} A_{i j}, \quad(i=1, \ldots, N) .
$$

In calculation of each influence coefficient $A_{i j}(j \neq i)$, OpenMP parallelization technique is employed to distribute the computation burden on multiple processors of a single computer. The parallelization works well since calculation of the influence coefficient on one element is independent from that on another element. After that, the Gauss elimination algorithm is used to solve the linear system, which is extremely robust regardless of arbitrary shape of the structure.

Given solution for the linear system, we can get the wave exciting force, added mass and added damping by directly integrating the corresponding hydrodynamic pressure over the immersed body surface, respectively, i.e.:

$$
f_{j}=\mathrm{i} \rho \omega \int_{S_{B}}\left(\phi_{0}+\phi_{4}\right) n_{j} d S,
$$

and:

$$
a_{i j}+\frac{\mathrm{i} b_{i j}}{\omega}=\rho \int_{S_{B}} \phi_{i} n_{j} d \mathrm{~S} .
$$

\subsection{Direct Calculation of Free-Surface Green's Function}

As pointed out in the introduction, accurate calculation of the free-surface Green function is of great importance to the final solution of the problem. At a preliminary step, we may apply the function 
decomposition method which was proposed by Newman [17]. Equation (10) can be decomposed into a simplified form:

$$
G=\ln \frac{r}{r_{1}}-2 F_{1}(x, z)-2 \pi \mathrm{i} e^{K(z+\zeta)} \cos K(x-\xi),
$$

where i denotes the imaginary unit. The singular part of Equation (23) is:

$$
F_{1}(x, z)=P V \int_{0}^{\infty} \frac{e^{\mu(z+\zeta)}}{\mu-K} \cos \mu(x-\xi) d \mu,
$$

where $P V$ denotes the Cauchy principle value of the integral. The principle task here is to evaluate the real function $F_{1}(x, z)$ for all relevant values of input parameters $(x, z)$ of possible physical interest [17]. Using the identity:

$$
P V \int_{0}^{2 K} \frac{d \mu}{\mu-K}=0
$$

Equation (24) can be written [18] in a more convenient form from the view point of numerical evaluation:

$$
F_{1}(x, z)=\int_{0}^{2 K} \frac{f_{1}(\mu)-f_{1}(K)}{\mu-K} d \mu+\int_{2 K}^{\infty} \frac{f_{1}(\mu)}{\mu-K} d \mu
$$

where:

$$
f_{1}(\mu)=e^{\mu(z+\zeta)} \cos \mu(x-\xi) .
$$

In the neighborhood of $\mu=K$, linear approximation may be applied such that:

$$
\frac{f_{1}(\mu)-f_{1}(K)}{\mu-K}=f_{1}^{\prime}(K)=e^{K(z+\zeta)}[(z+\zeta) \cos K(x-\xi)-(x-\xi) \sin K(x-\xi)] .
$$

Hence, Equation (25) can be evaluated accurately by an adaptive Gauss-Kronrod-type quadrature algorithm (see Appendix A).

Following a similar procedure, the derivatives of the Green function with respect to $x$ and $z$ can be normalized as:

$$
\begin{aligned}
& G_{x}=(x-\xi)\left(\frac{1}{r^{2}}-\frac{1}{r_{1}^{2}}\right)+2 K F_{2}(x, z)+2 \pi \mathrm{i} K e^{K(z+\zeta)} \sin K(x-\xi), \\
& G_{z}=\frac{(z-\zeta)}{r^{2}}-\frac{(z+\zeta)}{r_{1}^{2}}-2 K F_{3}(x, z)-2 \pi \mathrm{i} K e^{K(z+\zeta)} \cos K(x-\xi),
\end{aligned}
$$

where their singular parts are:

$$
\begin{aligned}
& F_{2}(x, z)=P V \int_{0}^{\infty} \frac{\mu e^{\mu(z+\zeta)}}{\mu-K} \sin \mu(x-\xi) d \mu, \\
& F_{3}(x, z)=P V \int_{0}^{\infty} \frac{\mu e^{\mu(z+\zeta)}}{\mu-K} \cos \mu(x-\xi) d \mu .
\end{aligned}
$$

Equations (30) and (31) can be formulated as the same form as Equation (25), where:

$$
\begin{gathered}
f_{2}(\mu)=\mu e^{\mu(z+\zeta)} \sin \mu(x-\xi), \\
f_{3}(\mu)=\mu e^{\mu(z+\zeta)} \cos \mu(x-\xi), \\
f_{2}^{\prime}(K)=e^{K(z+\zeta)}[\sin K(x-\xi)+K(z+\zeta) \sin K(x-\xi)+K(x-\xi) \cos K(x-\xi)], \\
f_{3}^{\prime}(K)=e^{K(z+\zeta)}[\cos K(x-\xi)+K(z+\zeta) \cos K(x-\xi)-K(x-\xi) \sin K(x-\xi)] .
\end{gathered}
$$




\subsection{Fast Evaluation by the Analytical Method}

Although calculation of the free-surface Green function becomes applicable following the method described in Section 2.3, a large amount of computation time would be consumed due to the direct integration by the meticulous adaptive numerical quadrature method. The reason for that is the effort of dealing with singularity in the denominator, as well as the oscillating inherence of the integrand. A possible way for its improvement is to derive an alternative analytical expression which can automatically remove the troublesome singularity, as described below.

Based on McIver [19], we can obtain the following representation for the principle value of the singular integral without too much of difficulty:

$$
P V \int_{0}^{\infty} \frac{e^{\mu(z+\zeta)+\mathrm{i} \mu X}}{\mu-K} \mathrm{~d} \mu= \begin{cases}e^{K(z+\zeta)+\mathrm{i} K X}\left(-\pi \mathrm{i}+E_{1}(K(z+\zeta)+\mathrm{i} K X)\right), & X<0,(z+\zeta) \leq 0 \\ -e^{K(z+\zeta)} E i(-K(z+\zeta)), & X=0,(z+\zeta) \leq 0 \\ e^{K(z+\zeta)+\mathrm{i} K X}\left(\pi \mathrm{i}+E_{1}(K(z+\zeta)+\mathrm{i} K X)\right), & X>0,(z+\zeta) \leq 0\end{cases}
$$

where the exponential integrals are defined as:

$$
\begin{gathered}
E i(x)=\int_{-\infty}^{x} \frac{e^{t}}{t} d t, \quad(x>0), \\
E_{1}(Z)=\int_{Z}^{\infty} \frac{e^{-t}}{t} d t, \quad(\arg |Z|<\pi) .
\end{gathered}
$$

Let $Z=K(z+\zeta)+i K X$, the real part of Equation (10) can then be written as:

$$
\operatorname{Re}\{G\}=\ln \frac{r}{r_{1}}-2 \begin{cases}\operatorname{Re}\left\{e^{Z}\left(-\pi \mathrm{i}+E_{1}(Z)\right)\right\}, & X<0,(z+\zeta) \leq 0 \\ \operatorname{Re}\left\{-e^{Z} E i(-K z)\right\}, & X=0,(z+\zeta) \leq 0 \\ \operatorname{Re}\left\{e^{Z}\left(\pi \mathrm{i}+E_{1}(Z)\right)\right\}, & X>0,(z+\zeta) \leq 0\end{cases}
$$

while the imaginary part is obtained by applying the residue theorem, after which we find:

$$
\operatorname{Im}\{G\}=-2 \mathrm{i} \pi \operatorname{Re}\left(e^{Z}\right) .
$$

Based on the following identities of the exponential integral, i.e.,:

$$
\begin{gathered}
\frac{\mathrm{d} E_{1}(Z)}{\mathrm{d} Z}=-\frac{e^{-Z}}{Z}, \\
\frac{\mathrm{d} E i(z)}{\mathrm{d} z}=\frac{e^{-z}}{z},
\end{gathered}
$$

It is possible to calculate derivatives of the Green function with respect to $x$ and $z$, in which their real parts are corresponding to:

$$
\begin{gathered}
\operatorname{Re}\left\{G_{x}\right\}=(x-\xi)\left(\frac{1}{r^{2}}-\frac{1}{r_{1}^{2}}\right)-2 \begin{cases}\operatorname{Re}\left\{\mathrm{i} K e^{Z}\left(-\pi \mathrm{i}+E_{1}(Z)\right)-\frac{\mathrm{i} K}{Z}\right\}, & X<0,(z+\zeta) \leq 0 \\
0, & X=0,(z+\zeta) \leq 0 \\
\operatorname{Re}\left\{\mathrm{i} K e^{Z}\left(\pi \mathrm{i}+E_{1}(Z)\right)-\frac{\mathrm{i} K}{Z}\right\}, & X>0,(z+\zeta) \leq 0\end{cases} \\
\operatorname{Re}\left\{G_{z}\right\}=\frac{(z-\zeta)}{r^{2}}-\frac{(z+\zeta)}{r_{1}^{2}}-2 \begin{cases}\operatorname{Re}\left\{K e^{Z}\left(-\pi \mathrm{i}+E_{1}(Z)\right)-\frac{K}{Z}\right\}, & X<0,(z+\zeta) \leq 0 \\
\operatorname{Re}\left\{-K e^{z} E i(-K z)+\frac{1}{K Z}\right\}, & X=0,(z+\zeta) \leq 0 \\
\operatorname{Re}\left\{e^{Z}\left(\pi \mathrm{i}+E_{1}(Z)\right)-\frac{K}{Z}\right\}, & X>0,(z+\zeta) \leq 0\end{cases}
\end{gathered}
$$


respectively. Their imaginary parts are easily obtained by applying the residue theorem as:

$$
\begin{aligned}
& \operatorname{Im}\left\{G_{x}\right\}=2 \mathrm{i} K \pi \operatorname{Im}\left(e^{Z}\right), \\
& \operatorname{Im}\left\{G_{z}\right\}=-2 \mathrm{i} K \pi \operatorname{Re}\left(e^{Z}\right) .
\end{aligned}
$$

Through this method, we are able to calculate the free-surface Green function in a fast manner, since Equations (39), (40), and (43)-(46) are all in analytical form, which just simply consists of the exponential functions and the trigonometric functions.

In terms of the following two coordinates:

$$
X=K(x-\xi), Y=K|z+\zeta|
$$

The three singular functions in Equations (24), (30) and (31) can be expressed as $F_{1}(X, Y), F_{2}(X, Y)$, and $F_{3}(X, Y)$. Figures $2-4$ show a comparison between plots of the three singular functions calculated by the direct integration method and the analytical solution method. In general, the two methods get almost same results which are hard to be distinguished from each other. It is obviously to see that $F_{1}(X, Y)$ and $F_{3}(X, Y)$ are even functions in symmetric with respect to the $Y$ axis, while $F_{2}(X, Y)$ is an odd function which is anti-symmetric about the $Y$ axis. Remarkable variations with a period of $\pi$ in parallel to the $X$ axis can be observed in all the plots for the region of $Y \in[0,3]$. It is also important to see that the variation becomes slow-varying with the increase of $Y$ in the region of $Y \in[3,9+]$.

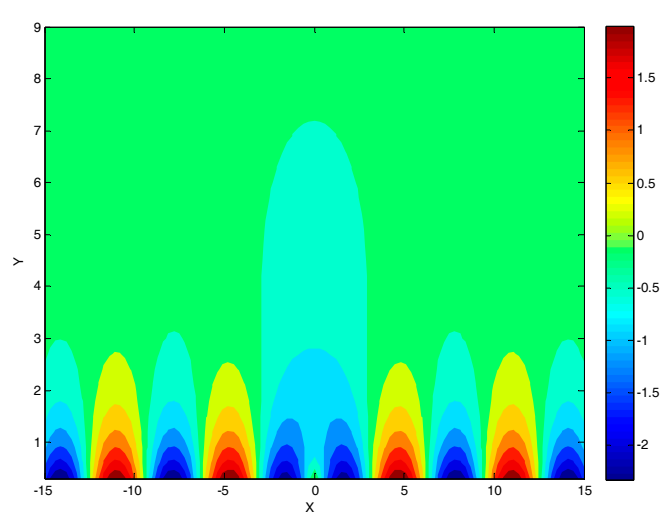

(a)

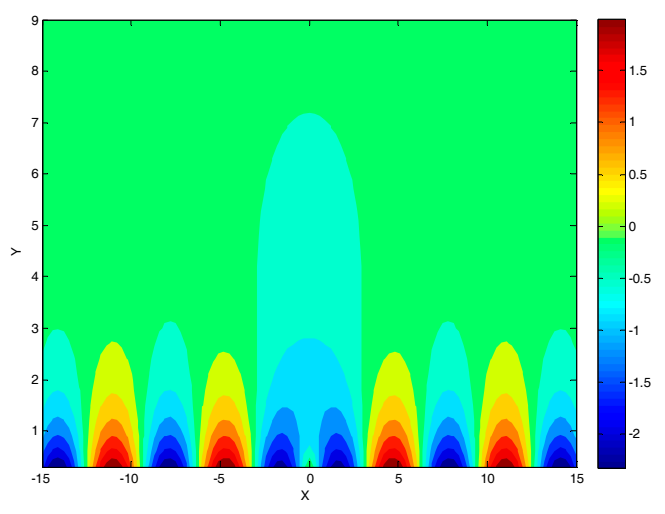

(c)

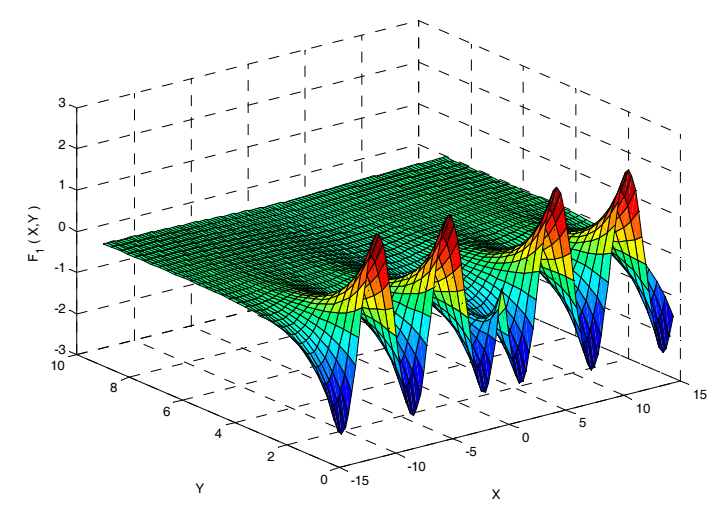

(b)

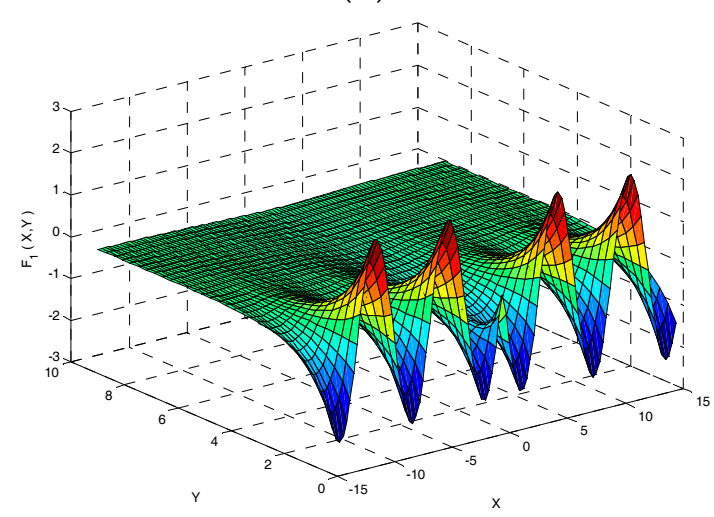

(d)

Figure 2. Comparison of the singular function $F_{1}(X, Y)$ calculated by the two methods: (a) contour plot by direct integration; (b) oblique view by direct integration; (c) contour plot by analytical solution; and (d) oblique view by analytical solution. 


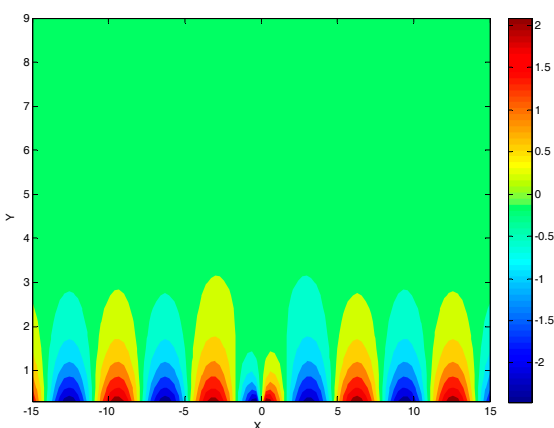

(a)

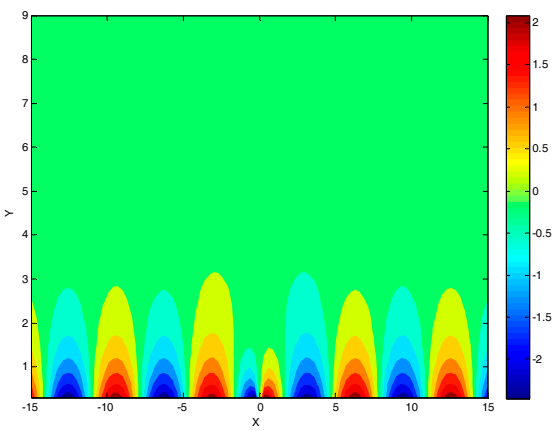

(c)

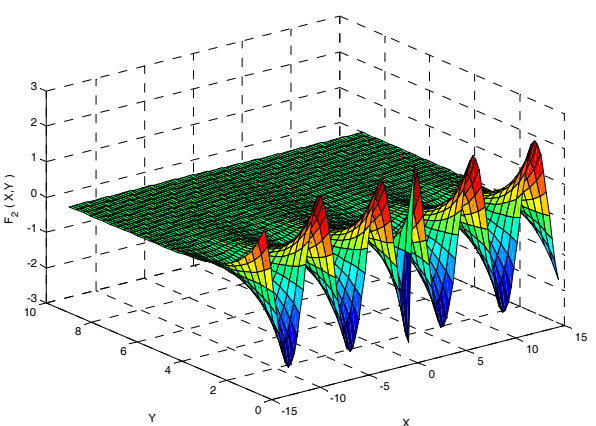

(b)

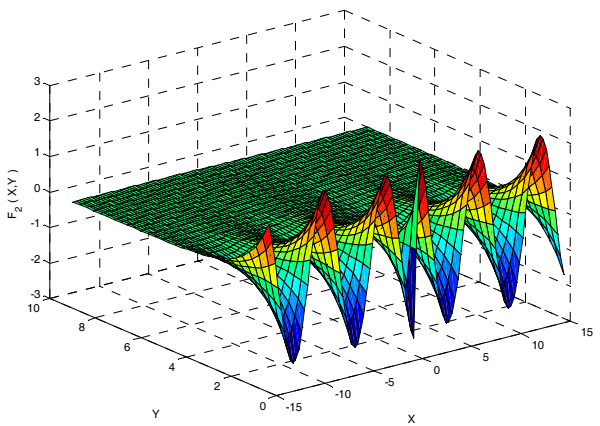

(d)

Figure 3. Comparison of the singular function $F_{2}(X, Y)$ calculated by the two methods. For captions of the subplots please refer to Figure 2.

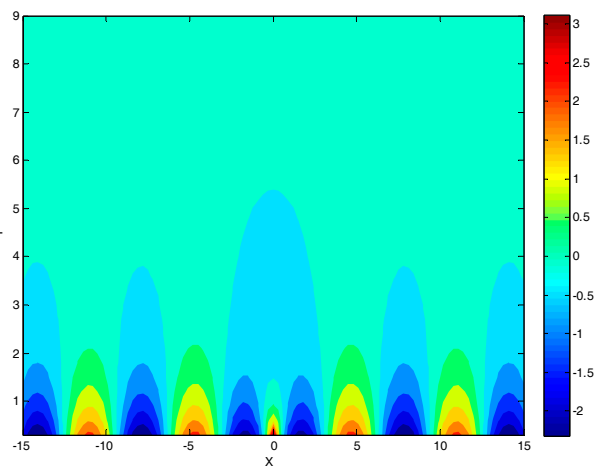

(a)

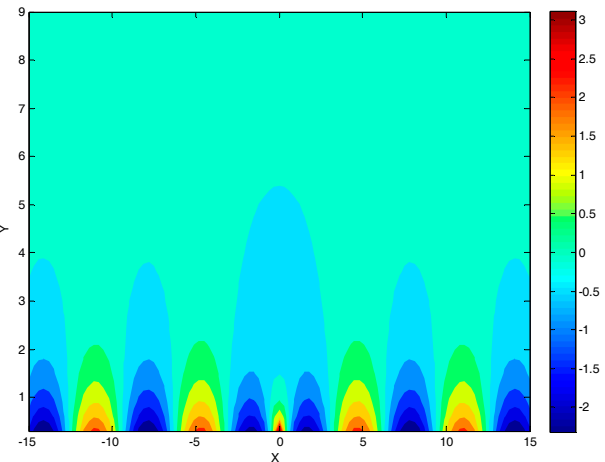

(c)

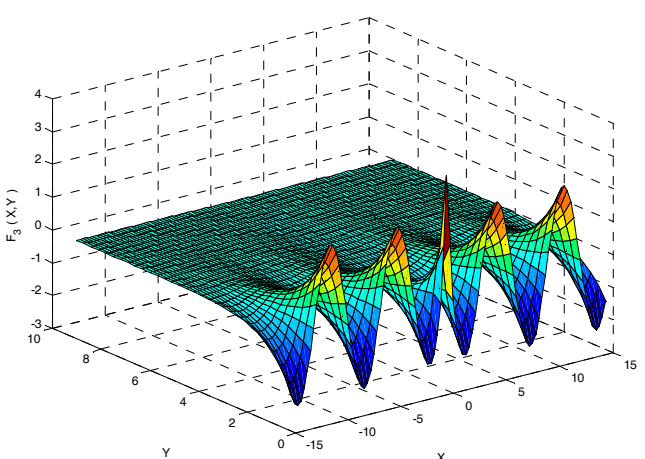

(b)

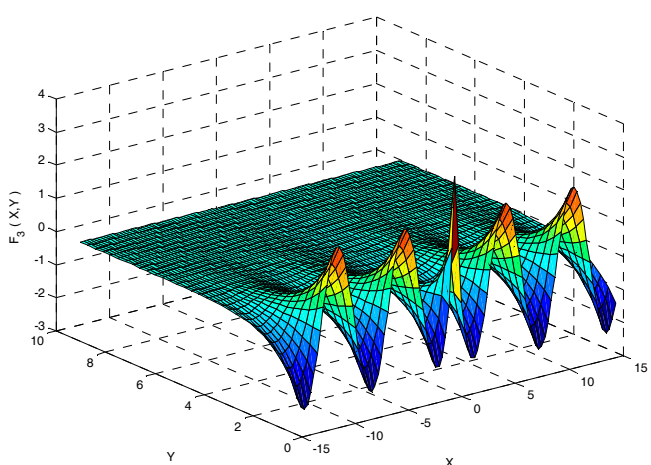

(d)

Figure 4. Comparison of the singular function $F_{3}(X, Y)$ calculated by the two methods. For captions of the subplots please refer to Figure 2. 


\section{Numerical Results and Discussion}

Based on the direct calculation method and the analytical solution method described above, values of the free-surface Green function and its derivatives are compared, as shown in Figures 5 and 6, against variation of the physical horizontal distance $|X-\xi|$ between the source and the field points. Both the real part and the imaginary part are compared, showing that they coincide fairly well with each other. It should be noted that calculation of the imaginary parts is relatively straightforward since the real parts contain troublesome principal values of the singular integrals.

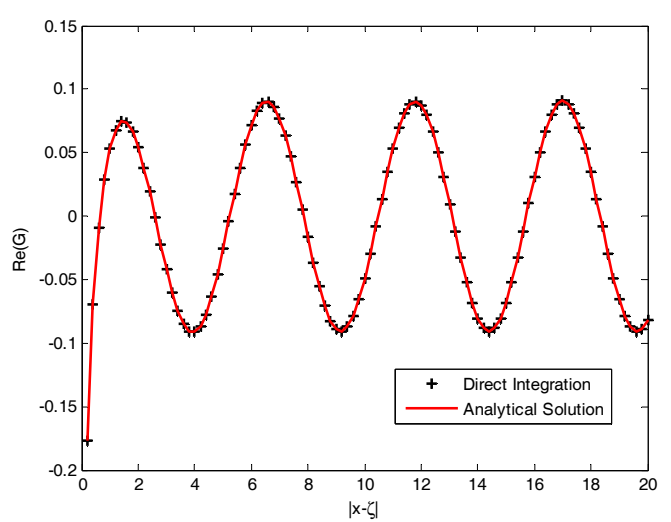

(a)

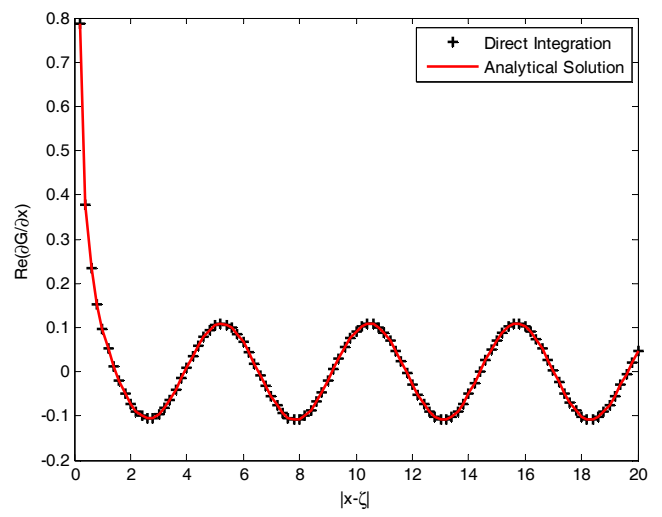

(c)

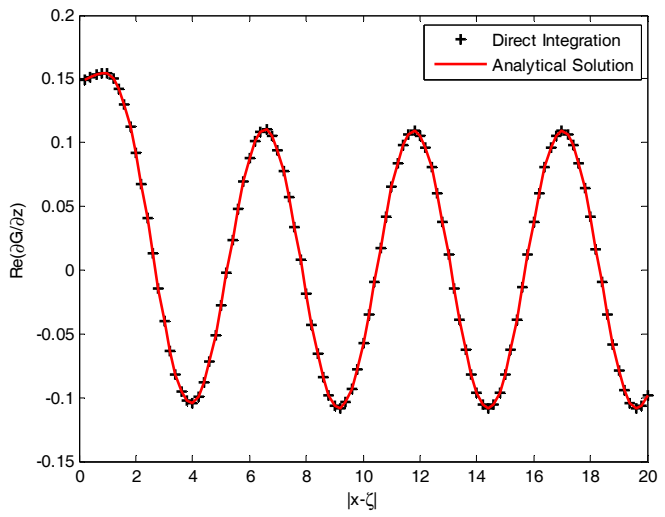

(e)

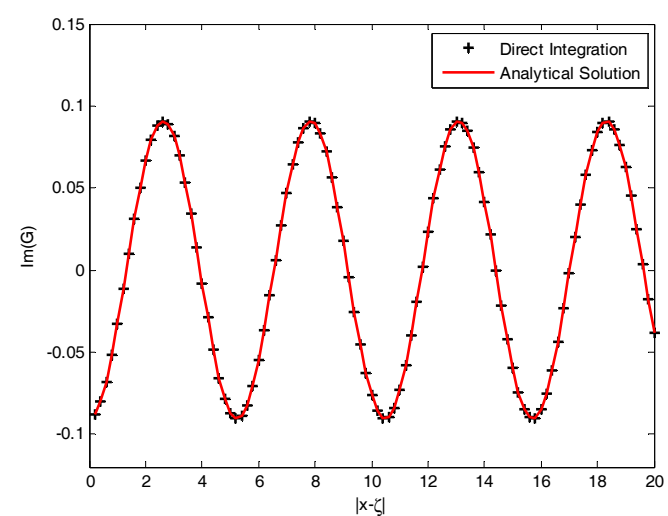

(b)

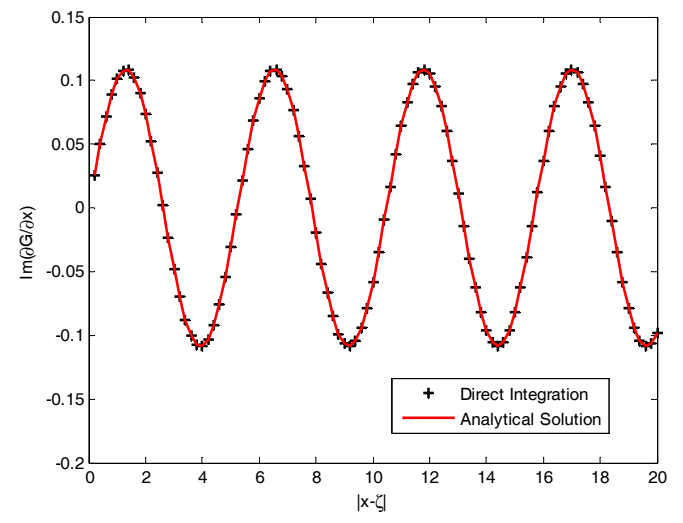

(d)

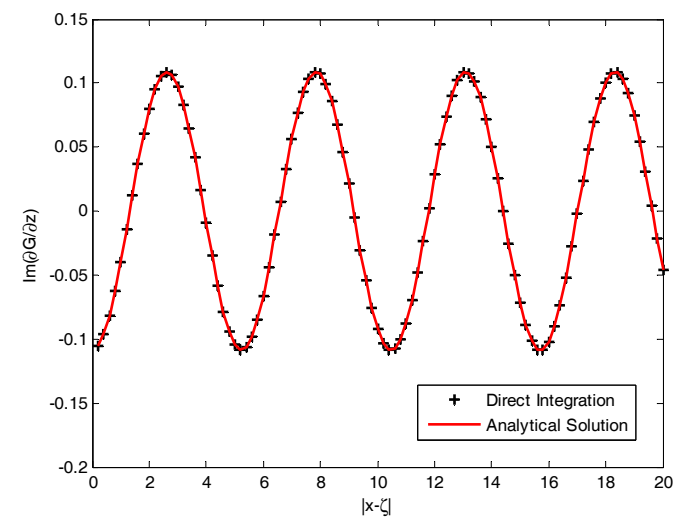

(f)

Figure 5. Comparison of two methods for calculating Wehausen's Green function and its derivatives $\left(K=1.2 \mathrm{~m}^{-1}, \zeta=-1.0 \mathrm{~m}, z=-1.0 \mathrm{~m}\right)$ : (a) real part value of $G$; (b) imaginary part value of $G$; (c) real part value of $G_{x} ;(\mathbf{d})$ imaginary part value of $G_{x} ;(\mathbf{e})$ real part value of $G_{z}$; and (f) imaginary part value of $G_{z}$. 


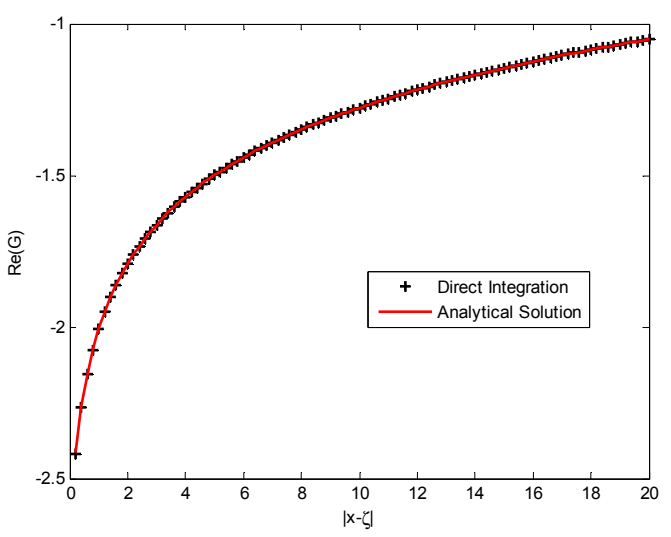

(a)

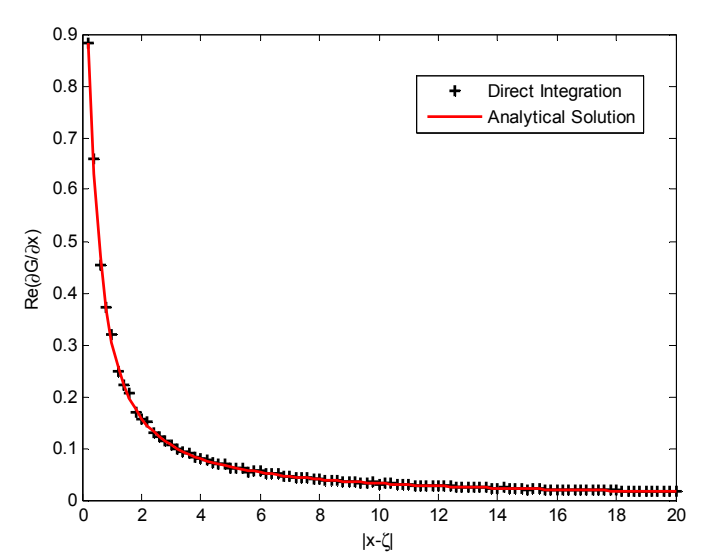

(c)

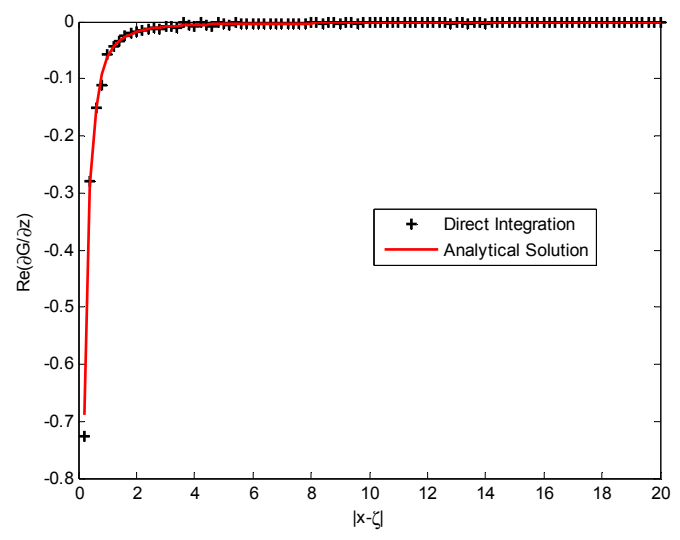

(e)

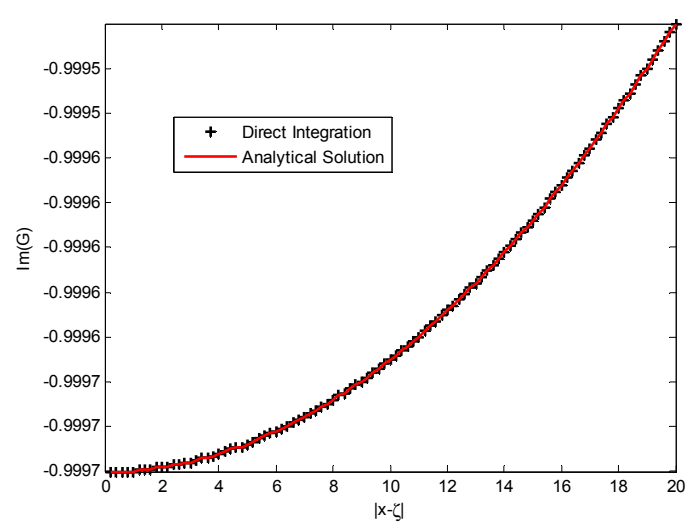

(b)

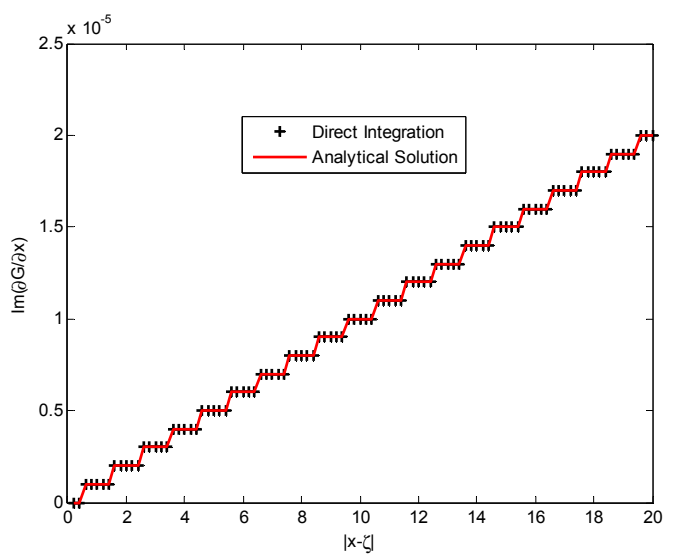

(d)

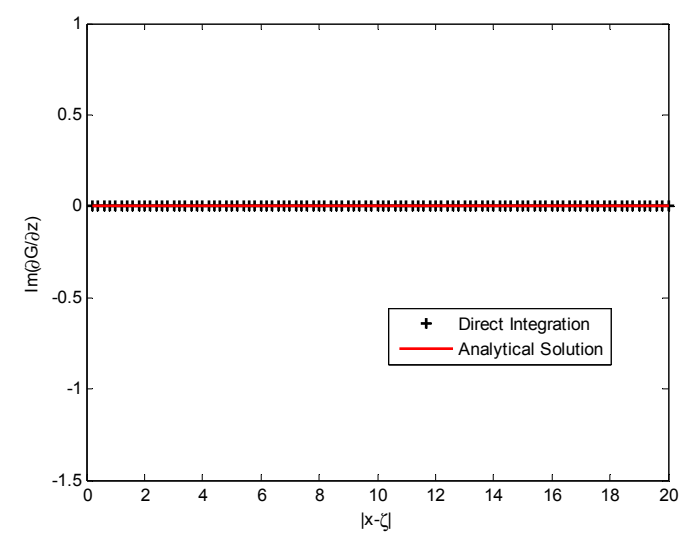

(f)

Figure 6. Comparison of two methods for calculating Wehausen's Green function and its derivatives $\left(K=0.001 \mathrm{~m}^{-1}, \zeta=-0.1 \mathrm{~m}, z=-0.2 \mathrm{~m}\right)$. For captions of the subplots please refer to Figure 5 .

Due to different nature of the free-surface Green function when the source point locates at the free surface or not, we need to verify the results for both floating bodies and submerged bodies. In order to compare with some existing analytical results, we select horizontal floating/submerged circular cylinders for the benchmark examples. When the cylinder is submerged, its wet body surface should be considered as a completely immersed circle; when it is floating with its centroid located on exactly the mean water surface, whereas its wet body surface should be treated as a half circle. Their analytical solutions are all obtained based on the so-called multipole expansion method, which were published in $[2,20]$ for a submerged circle in water of infinite depth, and in $[1,21]$ for a half circle in water of 
infinite depth. The RKG_BEM is also implemented for comparison, in which all of the boundaries should be taken into consideration; whereas for the FSG_BEM, only the body surface needs to be meshed. The computations are implemented on a SONY laptop (Sony Corporation, Tokyo, Japan), with an Intel(R) (Intel, Inc., Santa Clara, CA, USA) Core(TM) i7-2670QM CPU of 2.2 GHz, on 64-bit Windows operating system. The OpenMP parallelization technique has been applied in the parallel mode.

Figure 7 shows modulus of complex exciting force, added mass and added damping of a semi-immersed cylinder of radius a in comparison with those computed by the RKG_BEM and the analytical multipole expansion method [20]. The corresponding meshes used by the two boundary element methods are specified in Table 1. The computation takes $39.08 \mathrm{~s}$ for the RKG_BEM and $2.67 \mathrm{~s}$ for the FSG_BEM, both in parallel mode. In Figure 7, the present method based on the analytically evaluated free-surface Green function achieves good agreement with both of the other two methods. Noted that, there are some odd points on the curve calculated by the FSG_BEM. This phenomenon should be attributed to the so-called "irregular frequencies" $[10,22]$. The irregular frequencies are given by the eigenvalues associated with the interior Dirichlet problem, within the fluid domain on which the integral equation is applied. The discrete boundary integral equation is ill-conditioned and not uniquely solvable at these frequencies.

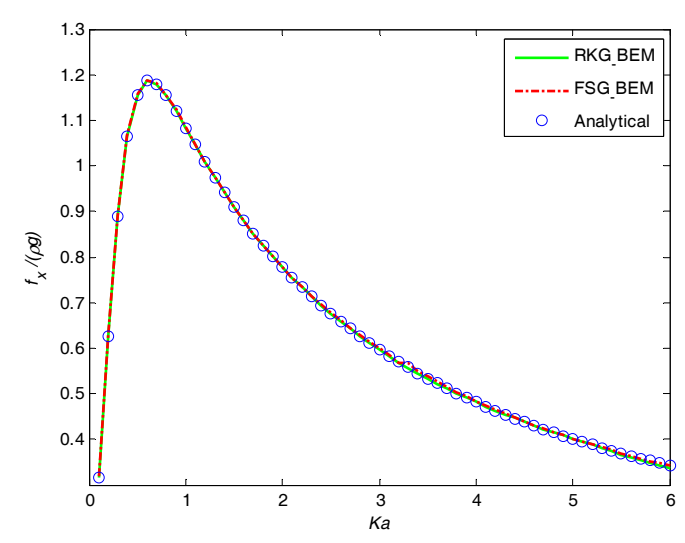

(a)

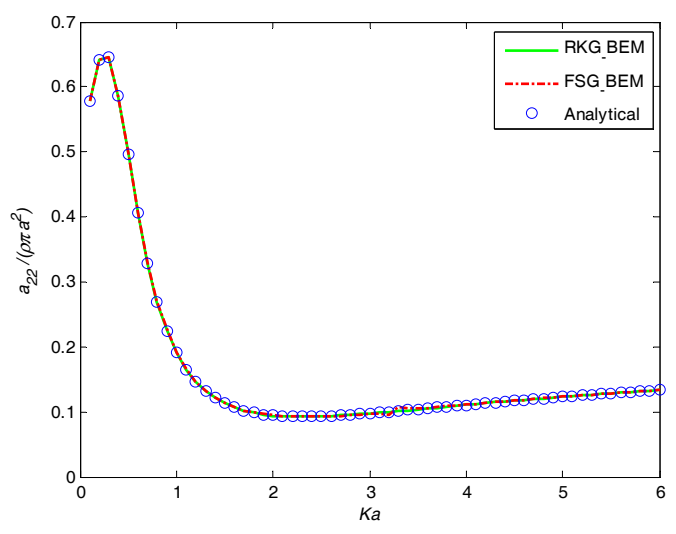

(c)

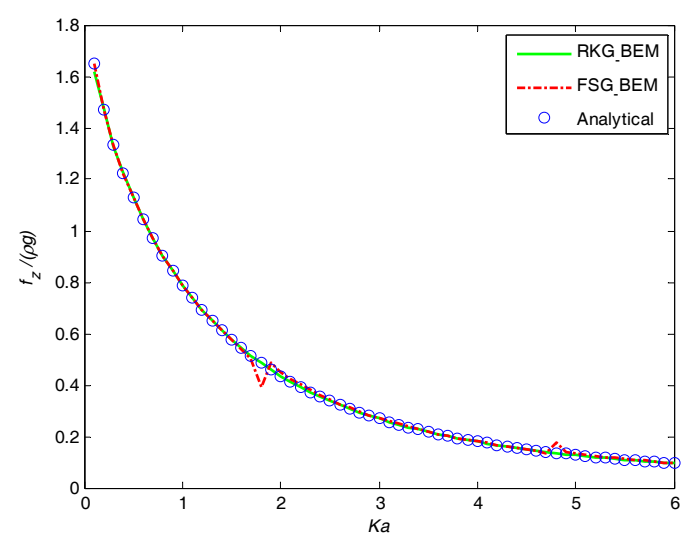

(b)

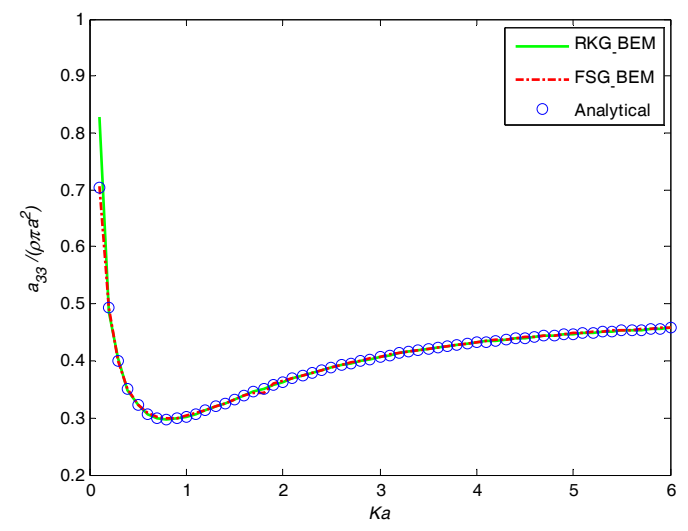

(d)

Figure 7. Cont. 


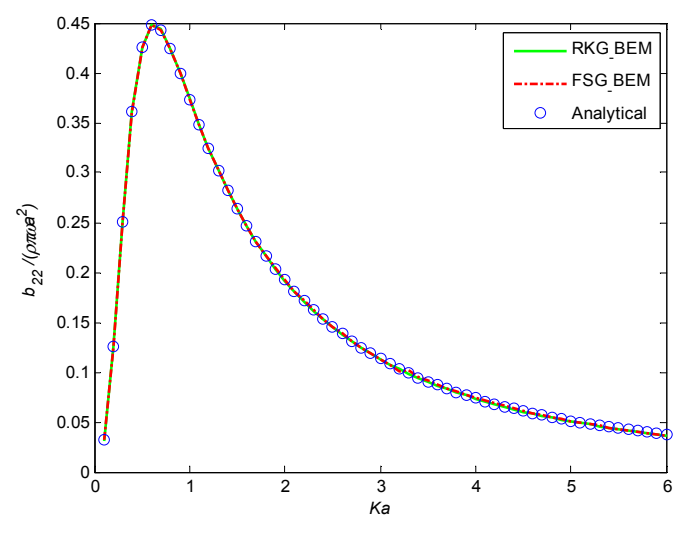

(e)

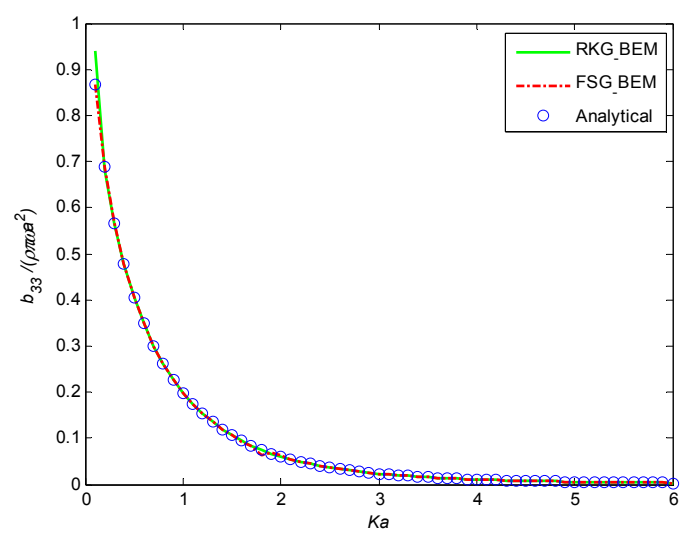

(f)

Figure 7. Comparison of hydrodynamic characteristics of a floating cylinder, in semi-immersed circle of radius a: (a) sway exciting force; (b) heave exciting force; (c) sway added mass; (d) heave added mass; (e) sway added damping; and (f) heave added damping.

Table 1. Mesh specifications for the case shown in Figure 4. $L_{\mathrm{F}}, L_{\mathrm{U}}, L_{\mathrm{L}}$ and $L_{\mathrm{B}}$ denote the length of the boundaries as shown in Figure 1, respectively, and $N_{\mathrm{F}}, N_{\mathrm{U}}, N_{\mathrm{L}}$, and $N_{\mathrm{B}}$ denote the number of elements meshed on the boundaries, respectively.

\begin{tabular}{lcccccccc}
\hline Method & $L_{\mathbf{F}}$ & $L_{\mathbf{U}}$ & $L_{\mathbf{L}}$ & $\boldsymbol{L}_{\mathbf{B}}$ & $N_{\mathbf{F}}$ & $N_{\mathbf{U}}$ & $N_{\mathbf{L}}$ & $N_{\mathbf{B}}$ \\
\hline FSG_BEM & $/$ & $/$ & $/$ & $\pi a$ & $/$ & $/$ & $/$ & 10 \\
RKG_BEM & $60 a$ & $20 a$ & $20 a$ & $\pi a$ & 240 & 90 & 90 & 30 \\
\hline
\end{tabular}

Figure 8 shows modulus of complex exciting forces, added mass and added damping of a submerged cylinder in comparison with those computed by the RKG_BEM and the analytical multipole expansion method [21]. The computation takes $49.21 \mathrm{~s}$ for the RKG_BEM and $2.65 \mathrm{~s}$ for the FSG_BEM, both in parallel mode. In the solution of multipole expansion method, we derive a new exact formulation (see Appendix B) for the multipole expansion coefficient $A_{m n}$ which is troublesome for calculation due to its high singularity in the integrand. In this case, the radius of the cylinder is a, and the submergence (vertical distance from its centroid to the mean free surface) is $f / a=1.5$. The meshes used by the two boundary element methods are specified in Table 2. In Figure 8, similar to the case of semi-circle, the present method based on the analytically evaluated free-surface Green function highly agrees with both of the other two methods. In addition, there is no "irregular frequencies" phenomenon, which proves the knowledge that for submerged bodies, the solution is always unique.

Table 2. Mesh specifications for the case shown in Figure 5. Refer to Table 1 for the notes of the symbols.

\begin{tabular}{lcccccccc}
\hline Method & $L_{\mathbf{F}}$ & $L_{\mathbf{U}}$ & $L_{\mathbf{L}}$ & $L_{\mathbf{B}}$ & $N_{\mathbf{F}}$ & $N_{\mathbf{U}}$ & $N_{\mathbf{L}}$ & $N_{\mathbf{B}}$ \\
\hline FSG_BEM & $/$ & $/$ & $/$ & $2 \pi a$ & $/$ & $/$ & $/$ & 10 \\
RKG_BEM & $60 a$ & $20 a$ & $20 a$ & $2 \pi a$ & 240 & 90 & 90 & 60 \\
\hline
\end{tabular}

Figure 9 shows a comparison of computation time (unit: s) for 60 incident wave periods between the BEMs (boundary element methods) based on the direct integration and the analytical solution of the free-surface Green function, in either sequential mode or parallel mode. In Figure 9, a remarkable trend of reduction in CPU time is shown by using the parallel mode, which tends to be more apparent with increasing number of the total elements, in both Figure 9a,b. On the other hand, the analytical-based Green function has saved a significant amount of computation time for the BEM analysis. Roughly speaking, it has improved the computation speed for around 27 36 times in the sequential mode, 
and 12 60 times in the parallel mode, in comparison to the direct-integration Green function method, depending on the number of total elements in the input mesh of geometry. The computation time is further compared in Figure 10, for each calling of the subroutine, involving calculation for both the value of Green's function and its derivatives. It is evidently shown that the direct integration method consumes much more time than the analytical solution method. In addition, the computation time tends to increase proportionally as the increase of the point distance $|x-\xi|$, which is different from that of the series expansion method as reported in the three-dimensional problem in [17,23-25].

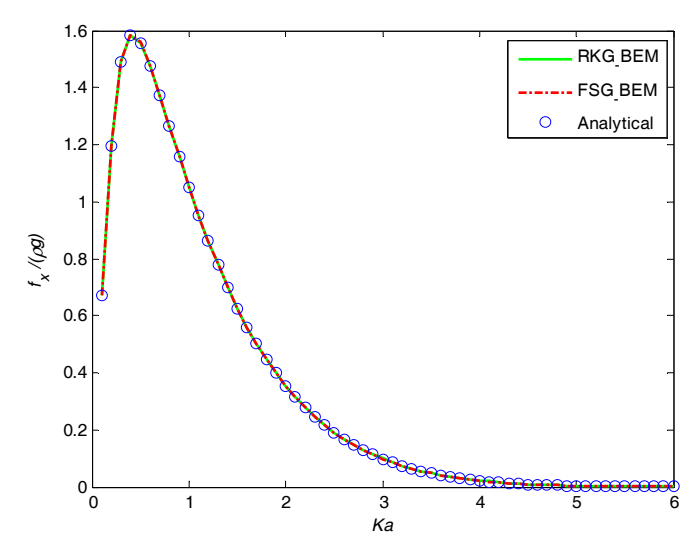

(a)

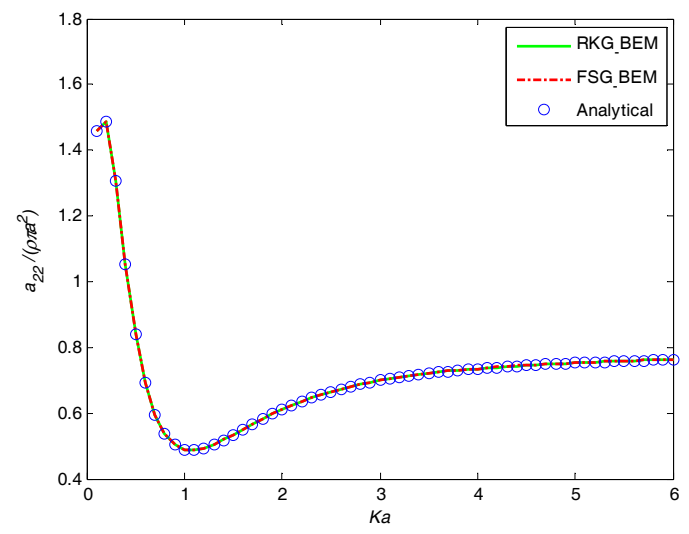

(c)

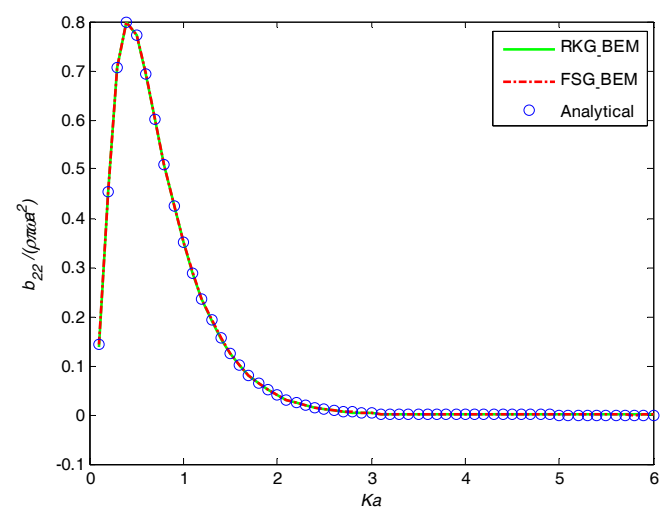

(e)

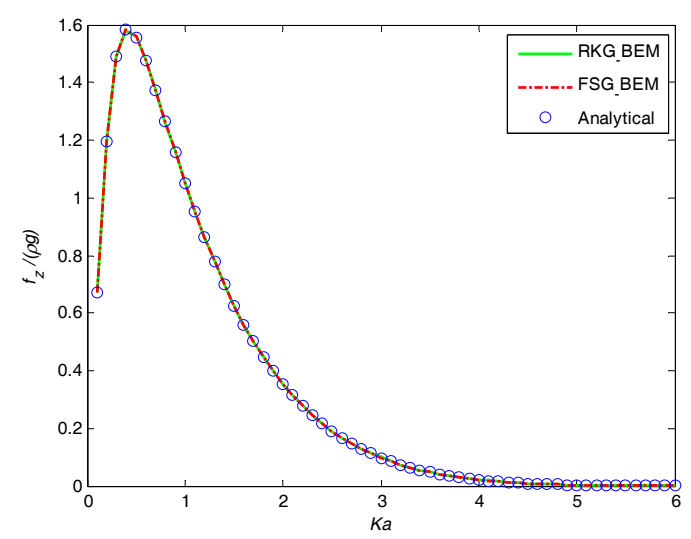

(b)

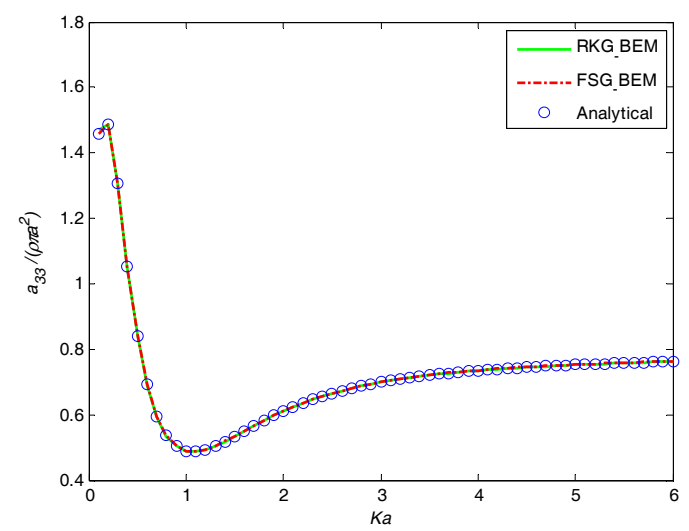

(d)

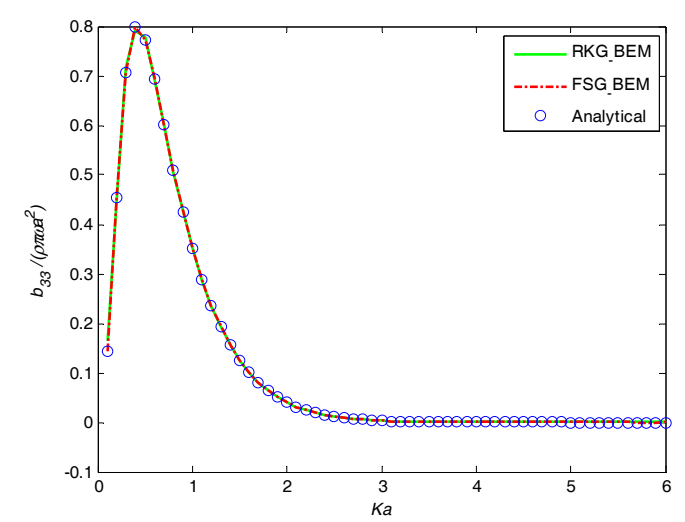

(f)

Figure 8. Comparison of hydrodynamic characteristics of a submerged cylinder. For captions of the subplots please refer to Figure 7. 


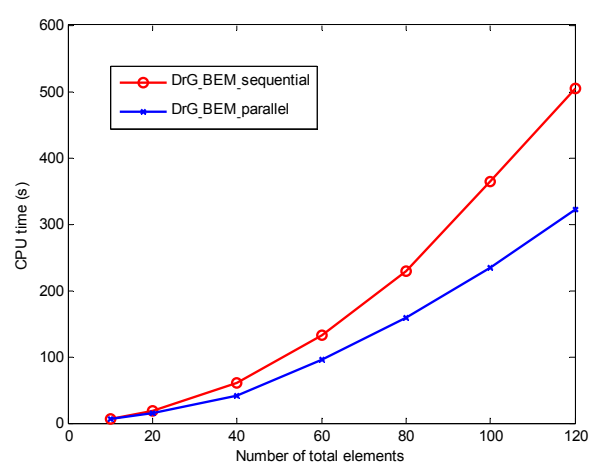

(a)

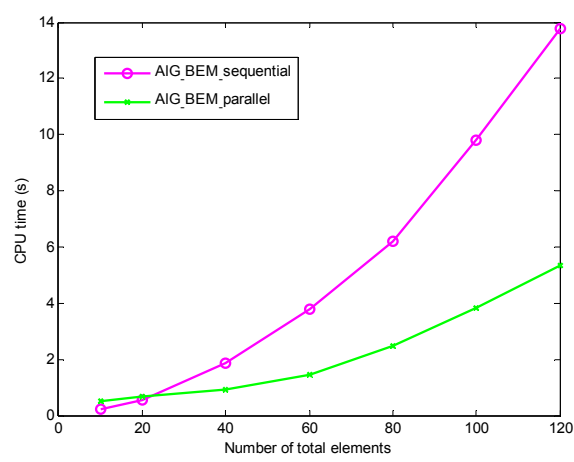

(b)

Figure 9. CPU time of the two boundary element methods based on different calculation schemes of the free-surface Green function in sequential or parallel mode: (a) comparison for the direct integration method; and (b) comparison for the analytical solution method.

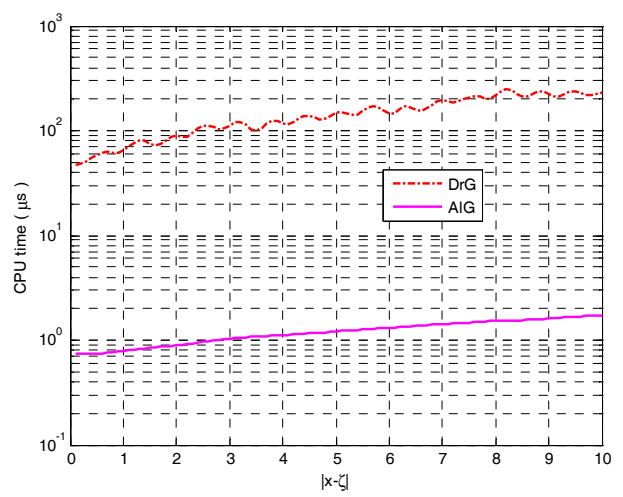

Figure 10. Computation time ( $\mu \mathrm{s})$ for each implementation of the Green's kernel, by the two methods, respectively, as a function of point distance $|x-\xi|$. The figure is obtained based on averaged CPU time of 1 million evaluations of the two codes, respectively, for every input of the wave period $\omega$.

Figure 11 demonstrates the convergence rate of the present FSG_BEM using analytical solution Green function. It is shown that with the increase of number of elements, the numerical solution gets close quickly to the exact solution [21], especially from 30 to 60 elements in the implemented case the difference is almost negligible. This may suggest that, for such cross-sections in simple geometries, only a few elements are sufficient to obtain a high accuracy.

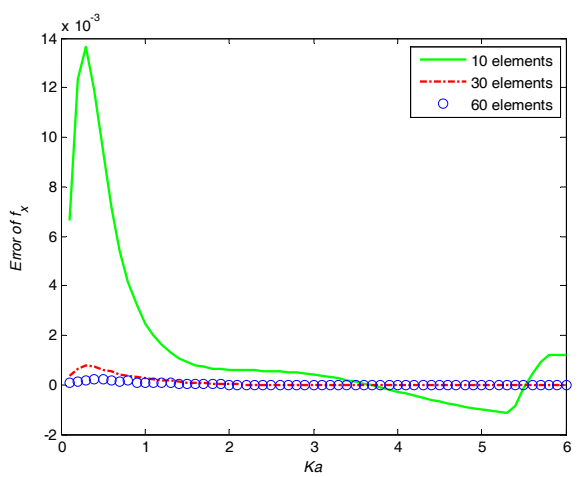

(a)

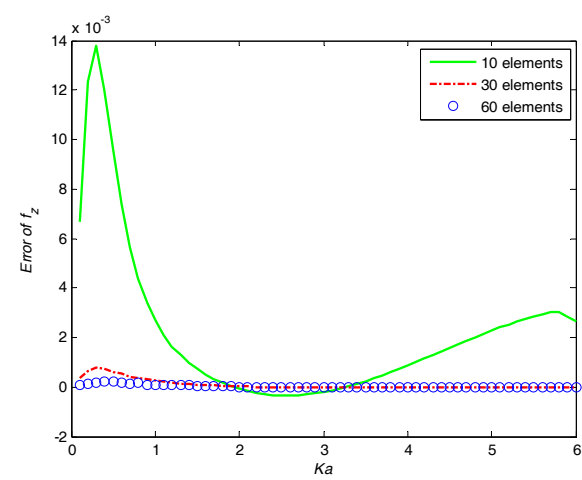

(b)

Figure 11. Convergence test of the present method to the exact solution [21] with respect to the number of elements: (a) sway exciting force; (b) heave exciting force. 
To further demonstrate the proposed FSG_BEM, wave interactions with a horizontal rectangular cylinder have been examined, for either floating or submerged cases. For simplicity, both of width and length of the rectangular cross-section shape are taken as $2 a$, respectively. $f / a$ is the submergence of its centroid, as defined in the circular cylinder case. We examine four different submergences (particularly, $f / a=0.0$ stands for the floating cylinder), and the results are shown in Figure 12. From the results, it is apparent that the floating cylinder is very different from those submerged in the water, especially in the low frequency region. In general, the peak value of the forces and its corresponding peak frequency tend to decrease as the submergence increases.

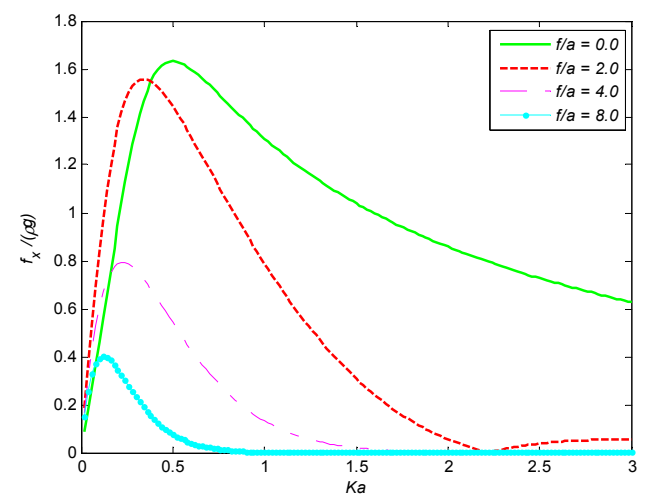

(a)

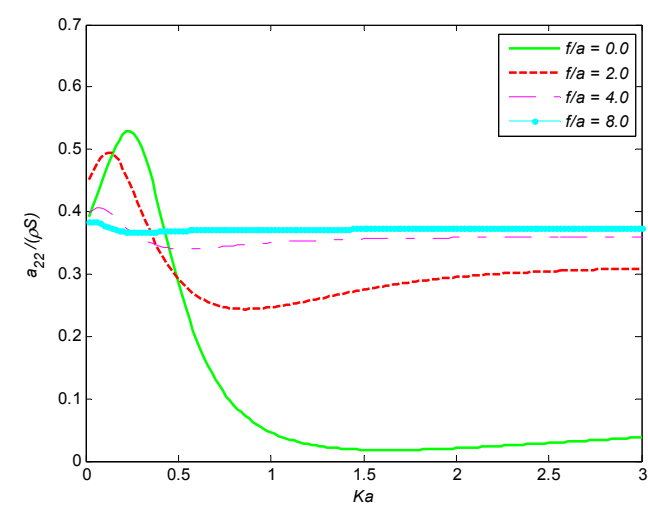

(c)

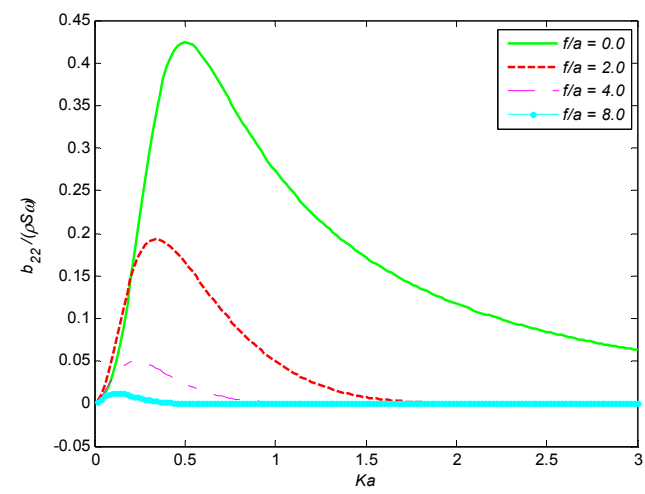

(e)

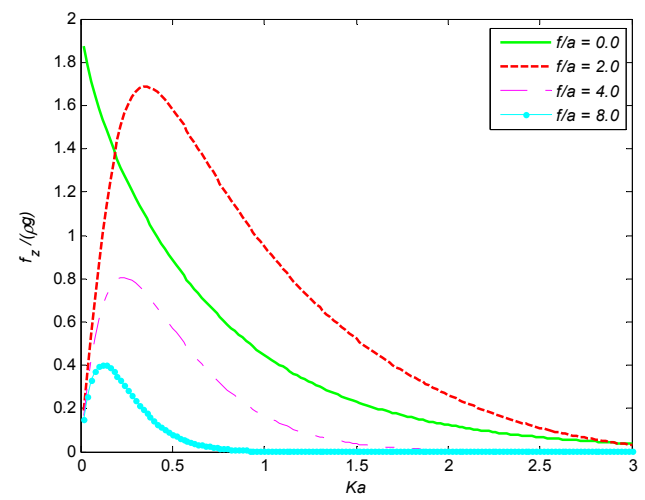

(b)

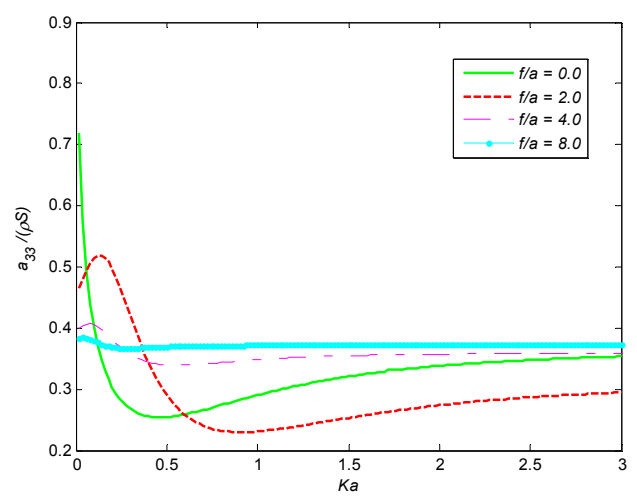

(d)

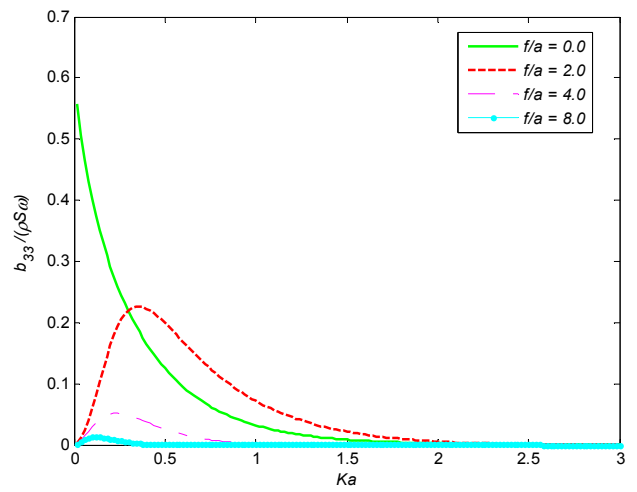

(f)

Figure 12. Variation of hydrodynamic characteristics of a floating rectangular cylinder $(f / a=0.0)$ and submerged rectangular cylinder for different submergences $(f / a=2.0, f / a=4.0$ and $f / a=8.0)$. $S$ represents the cross-section area (note that $S$ of a floating rectangular cylinder is half of a submerged one). For captions of the subplots please refer to Figure 7. 


\section{Conclusions}

In this paper, we presented a FSG_BEM, which applies a three-node higher-order scheme, and an analytical algorithm of the free-surface Green function. Various numerical results show that the present method deserves high accuracy, good convergence, and fast computation efficiency.

Acknowledgments: The authors gratefully acknowledge the financial support provided by National Natural Science Foundation of China (Grant No. 11072052 and No. 51221961), and the financial support provided by the National Basic Research Program of China (973-Program) (Grant No. 2011CB013703). The authors gratefully appreciate Phil McIver of Loughborough University, Maureen McIver of Loughborough University, and R. Porter of Bristol University, for their most valuable suggestions on the issue of removing the singularity.

Author Contributions: Yingyi Liu developed the algorithm of free-surface Green's function, performed all the calculations in the paper and wrote the manuscript. Ying Gou was the assistant supervisor of Yingyi Liu's Master study in DUT, who provided guidance, did proofreading and polished the English of this manuscript. Bin Teng was the supervisor of Yingyi Liu's Master study in DUT, who provided guidance and developed the two-dimensional HOBEM code used in this manuscript. Shigeo Yoshida is Yingyi Liu's current supervisor for postdoctoral research, who supplies salary and freedom for Yingyi Liu's research, including summarization of this work. All authors have read and approved the final manuscript.

Conflicts of Interest: The authors declare no conflict of interest.

\section{Abbreviations}

RKG_BEM Rankine Green function based Boundary Element Method

FSG_BEM Free-surface Green function based Boundary Element Method

DrG_BEM Boundary Element Method based on direct integration of the free-surface Green function

AlG_BEM Boundary Element Method based on analytical solution of the free-surface Green function

\section{Appendix A}

Let $[a, b]$ be the integration interval, $f$ be a Riemann integrable function, the following target integral:

$$
I=\int_{a}^{b} f(x) d x
$$

can be approximated by adding $n+1$ Kronrod points to the $n$-point Gauss quadrature rule, so that the function values produced by the lower-order rule can be re-used. This formula is called as Kronrod extension of Gaussian rules [26], which has a maximum degree of exactness $3 n+1$, i.e.,:

$$
I=\sum_{i=1}^{n} w_{i} f\left(x_{i}\right)+\sum_{k=1}^{n+1} w_{k}^{*} f\left(x_{k}^{*}\right), f \in P_{3 n+1},
$$

where $x_{i}$ are the Gauss nodes and $\omega_{i}$ the corresponding weights, $x_{k}^{*}$ and $\omega_{k}^{*}$ denote the Kronrod nodes and corresponding weights, respectively.

The difference between a Gauss quadrature rule and its Kronrod extension are often used as an estimate of the approximation error suggested by Piessens et al. [27]:

$$
\varepsilon=\left(200\left|G_{n}[a, b]-K_{2 n+1}[a, b]\right|\right)^{1.5},
$$

where $G_{n}$ represents the approximation of the initial Gaussian rule, and $K_{2 n+1}$ the approximation of its Kronrod extension.

Using the Gauss-Kronrod rule, an adaptive integral algorithm can be developed [28]:

Step 1. Firstly, use $n$-point Gauss rule and $(2 n+1)$-point Gauss-Kronrod rule to integrate $f(x)$ on the interval $[a, b]$, respectively. Two approximations of the integral, i.e., $G_{n}$ and $K_{2 n+1}$, will be obtained, as well as Equation (A3). If the error estimation is smaller than a prescribed tolerance Eps, the more accurate approximation $K_{2 n+1}$ is accepted as the final integral value; otherwise, go to Step 2. 
Step 2. Divide interval $[a, b]$ into two equal parts, i.e., $[a, m]$ and $[m, b]$, where $m=(a+b) / 2$, and compute the two sub-integrals independently:

$$
I=\int_{a}^{m} f(x) d x+\int_{m}^{b} f(x) d x .
$$

Again, the two respective approximations $G_{n}^{i}$ and $K_{2 n+1}^{i}$, as well as the local error estimate $\varepsilon^{i}$ will be attained:

$$
\varepsilon^{i}=\left(200\left|G_{n}^{i}[a, b]-K_{2 n+1}^{i}[a, b]\right|\right)^{1.5},
$$

where the superscript denotes the $i$ th sub-interval. If $\varepsilon^{i}$ is smaller than Eps, accept $K_{2 n+1}^{i}$ as the final integral value on $i$ th sub-interval, and stop the circulation; if not, continue to subdivide the sub-intervals and repeat Step 2.

\section{Appendix B}

In the multipole expansion method, Linton and McIver [20] gives an expression for wave scattering potential of a submerged horizontal cylinder in series form:

$$
\phi=\sum_{n=1}^{\infty} a^{n+1} \alpha_{n} \phi_{n}
$$

where:

$$
\begin{gathered}
\phi_{n}=\frac{e^{-\mathrm{i} n \theta}}{r^{n}}+\sum_{m=0}^{\infty} A_{m n} r^{m} e^{-\mathrm{i} m \theta}, \\
A_{m n}=\frac{(-1)^{m+n}}{m !(n-1) !} \oint_{0}^{\infty} \frac{\mu+K}{\mu-K} \mu^{m+n-1} e^{2 \mu \zeta} d \mu,
\end{gathered}
$$

where $a$ is the radius and $\zeta$ the submergence. Calculation of the multipole expansion coefficients $A_{m n}$ is not a trivial task, since usually a direct integration method will be adopted which leads to some substantial numerical errors. By the Newton's binomial theorem and through integration by parts, we derive the following series representations for its accurate calculation:

$$
A_{m n}=\operatorname{Re}\left(A_{m n}\right)+\mathrm{i} I m\left(A_{m n}\right),
$$

where:

$$
\begin{aligned}
& \operatorname{Re}\left(A_{m n}\right)=\frac{(-1)^{m+n}}{m !(n-1) !}\left\{\left(-\frac{1}{2 \zeta}\right)^{m+n}(m+n-1) !+2 K e^{2 K \zeta} .\right. \\
& \left.\left[\sum_{i=0}^{m+n-3} \frac{(m+n-1) !}{(m+n-1-i) i !} \frac{K^{i} e^{-2 K \zeta}}{(-2 \zeta)^{m+n-1-i}}\left(1+\sum_{j=1}^{m+n-2-i} \frac{(2 K \zeta)^{j}}{j !}\right)-\frac{(m+n-1)}{2 \zeta} K^{m+n-2} e^{-2 K \zeta}-K^{m+n-1} E i(-2 K \zeta)\right]\right\}
\end{aligned}
$$

and:

$$
\operatorname{Im}\left(A_{m n}\right)=\frac{(-1)^{m+n}}{m !(n-1) !} 2 \pi K^{m+n} e^{2 K \zeta}
$$

where $m \geq 0, n \geq 1, m, n \in Z^{*}$.

\section{References}

1. Ursell, F. On the heaving motion of a circular cylinder on the surface of a fluid. Q. J. Mech. Appl. Math. 1949, 2, 218-231. [CrossRef]

2. Ursell, F. Surface waves on deep water in the presence of a submerged circular cylinder I. In Mathematical Proceedings of the Cambridge Philosophical Society; Cambridge University Press: Cambridge, UK, 1950; Volume 46, pp. 141-158. 
3. Evans, D.V.; Linton, C.M. Active devices for the reduction in wave intensity. Appl. Ocean Res. 1989, 11, $26-32$. [CrossRef]

4. Bai, K.J. Diffraction of oblique waves by an infinite cylinder. J. Fluid Mech. 1975, 68, 513-535. [CrossRef]

5. Garrison, C.J. Interaction of oblique waves with an infinite cylinder. Appl. Ocean Res. 1984, 6, 4-15. [CrossRef]

6. Politis, C.G.; Papalexandris, M.V.; Athanassoulis, G.A. A boundary integral equation method for oblique water-wave scattering by cylinders governed by the modified Helmholtz equation. Appl. Ocean Res. 1984, 24, 215-233. [CrossRef]

7. Zheng, Y.H.; Shen, Y.M.; Ng, C.O. Effective boundary element method for the interaction of oblique waves with long prismatic structures in water of finite depth. Ocean Eng. 2008, 35, 494-502. [CrossRef]

8. Dean, R.G.; Ursell, F. Interaction of a Fixed, Semi-Immersed Circular Cylinder with a Train of Surface Waves; Technical Report No. 37; Hydrodynamic Lab, MIT: Cambridge, MA, USA, 1959.

9. Martin, P.A.; Dixon, A.G. The scattering of regular surface waves by a fixed, half-immersed, circular cylinder. Appl. Ocean Res. 1983, 5, 13-23. [CrossRef]

10. Wehausen, J.V.; Laitone, E.V. Surface waves. In Encyclopedia of Physics; Springer: Berlin, Germany, 1964; Volume 9, pp. 446-778.

11. Haskind, M.D. The diffraction of waves about a moving cylindrical vessel. Prikl. Mat. Mikh. 1953, 17, 431-442.

12. Newman, J.N. The approximation of free-surface Green functions. In Wave Asymptotics; Martin, P.A., Wickham, G.R., Eds.; Cambridge University Press: Cambridge, UK, 1992; pp. 107-135.

13. Noblesse, F. The Green function in the theory of radiation and diffraction of regular water waves by a body. J. Eng. Math. 1982, 16, 137-169. [CrossRef]

14. Telste, J.G.; Noblesse, F. Numerical evaluation of the Green Function of water-wave radiation and diffraction. J. Ship Res. 1986, 30, 69-84.

15. Ponizy, B.; Noblesse, F.; Ba, M.; Guilbaud, M. Numerical evaluation of free-surface Green functions. J. Ship Res. 1994, 38, 193-202.

16. Newman, J.N. An expansion of the oscillatory source potential. Appl. Ocean Res. 1984, 6, 116-117. [CrossRef]

17. Newman, J.N. Algorithms for free-surface green's function. J. Eng. Math. 1985, 19, 57-67. [CrossRef]

18. Monacella, V.J. The distribution due to a slender ship oscillating in a fluid of finite depth. J. Ship Res. 1966, 10, 242-252.

19. McIver, M. An example of non-uniqueness in the two-dimensional linear water wave problem. J. Fluid Mech. 1996, 315, 257-266. [CrossRef]

20. Linton, C.M.; McIver, P. Handbook of Mathematical Techniques for Wave/Structure Interactions; CRC Press: Boca Raton, FL, USA, 2001.

21. Yan, B.; Bai, W.; Liu, Y.; Gou, Y.; Ning, D. An analytical investigation on floating cylindrical breakwaters with different constraints. Ocean Eng. 2016. (submitted).

22. Taylor, R.E.; Zietsman, J. A comparison of localized finite element formulations for two-dimensional wave diffraction and radiation problems. Int. J. Numer. Methods Eng. 1981, 17, 1355-1384. [CrossRef]

23. Li, L. Numerical Seakeeping Predictions of Shallow Water Effect on Two Ship Interactions in Waves. Ph.D. Thesis, Dalhousie University, Halifax, NS, Canada, 2001.

24. Liu, Y.; Hu, C.; Sueyoshi, M.; Iwashita, H.; Kashiwagi, M. Motion response prediction by hybrid panel-stick models for a semi-submersible with bracings. J. Mar. Sci. Technol. 2016. [CrossRef]

25. Liu, Y.; Shigeo, Y. FinGreen3D: A Fortran code for efficiently computing free-surface Green's kernel in finite ocean depth with application to wave-structure interaction problems by using a satisfying region-decomposition strategy. Comput. Phys. Commun. 2016. (submitted).

26. Kronrod, A.S. Nodes and Weights of Quadrature Formulas-Sixteen-Place Tables: Authorized Translation from the Russian; Consultants Bureau: New York, NY, USA, 1965. 
27. Piessens, R.; de Doncker-Kapenga, E.; Uberhuber, C.W.; Kahaner, D.K. Quadpack: A Subroutine Package for Automatic Integration; Springer: Berlin/Heidelberg, Germany, 1983.

28. Gander, W.; Gautschi, W. Adaptive Quadrature-Revisited; Technical Report 306; Department of Computer Science, ETH Zurich: Zurich, Switzerland, 1998.

(c)

(C) 2016 by the authors; licensee MDPI, Basel, Switzerland. This article is an open access article distributed under the terms and conditions of the Creative Commons Attribution (CC-BY) license (http://creativecommons.org/licenses/by/4.0/). 